\title{
Molecular evolution of the carboxy terminal region of the zona pellucida 3 glycoprotein in murine rodents
}

\author{
Christine A Swann ${ }^{1,3}$, Steven J B Cooper ${ }^{2,3}$ and William G Breed ${ }^{1,3}$ \\ ${ }^{1}$ School of Medical Sciences, Faculty of Health Sciences, University of Adelaide, North Terrace, Adelaide, 5005 South \\ Australia, ${ }^{2}$ Evolutionary Biology Unit, South Australian Museum, Adelaide, South Australia and ${ }^{3}$ Centre for \\ Evolutionary Biology and Biodiversity, University of Adelaide, Adelaide, South Australia
}

Correspondence should be addressed to W G Breed; Email: bill.breed@adelaide.edu.au

\begin{abstract}
In mammals, before fertilization can occur, sperm have to bind to, and penetrate, the zona pellucida (ZP). In the laboratory mouse, which has been used as a model system for fertilization studies, sperm-ZP binding has been found to be mediated by a region at the carboxy terminal, encoded by exon 7 of the Zp3 gene. This region shows considerable interspecific sequence diversity with some evidence of adaptive evolution in mammals, suggesting that it may contribute to species-specific sperm-ZP binding. However, in a previous study of sequence diversity of ZP3 of three species of Australian murine rodents, we found an identical protein sequence of the region encoded by exon 7 . Here, we expand this earlier study to determine the sequence diversity of this region in 68 out of the 130 species of Australasian murine rodents. Maximum likelihood analyses, using representatives of both New Guinean and Australian taxa, provide evidence of positive selection at three codons adjacent to, or within, the putative combining-site for sperm of ZP3, but this was not evident when the analysis was restricted to the Australian taxa. The latter group showed low levels of both intra- and inter-generic sequence divergences in the region encoded by exon 7 of Zp3, with little evidence that this region contributes to species specificity of sperm-ZP binding. These findings suggest that the selective forces acting on the Zp3 exon 7 region during the evolution of the Australasian murine rodents have been variable, and that positive selection has only occurred in a few lineages.
\end{abstract}

Reproduction (2007) 133 697-708

\section{Introduction}

The mammalian zona pellucida (ZP) is an extracellular glycoprotein matrix that surrounds the ovulated oocyte through which the spermatozoon has to pass before it can bind to, and fuse with, the cell membrane of the oocyte (Yanagimachi 1994). The ZP is largely composed of three or four glycoproteins (ZP1-4), and a model based on studies of the ZP of the laboratory mouse (Mus musculus, 'the mouse'), has proposed that it is composed of filaments of alternating units of ZP2 and ZP3 crosslinked together by ZP1 (for reviews, see Green 1997, Wassarman 1999). A fourth ZP gene (Zp4) has been found to be expressed in the oocytes of the laboratory rat (Rattus norvegicus, 'the rat'), but there is no evidence that a functional $Z p 4$ gene exists in the mouse (Conner et al. 2005). In the mouse, ZP3 (mZP3) is the smallest of the three glycoproteins and appears to be important, not only as a structural component of the matrix (Rankin et al. 1996), but also as the primary binding site for the spermatozoon and for the induction of the acrosome reaction (Bleil \& Wassarman 1983).
Based on results from exon swapping and sitedirected mutagenesis experiments (Kinloch et al. 1995, Chen et al. 1998), it has been suggested that the region encoded by exon 7 , at the carboxyl terminus of the ZP3 glycoprotein, is the primary binding site for the spermatozoon (Rosiere \& Wassarman 1992, Kinloch et al. 1995). Within this region is a short stretch of amino acids (mZP3 ${ }_{328-343 \text {, the }}$ so-called 'combining-site for sperm') containing a series of serine residues (Ser-329, Ser-331 to Ser334), of which Ser-332 and Ser-334 are thought to provide $O$-linked glycosylation sites for primary sperm-ZP binding (Chen et al. 1998). By comparing this region of ZP3 of the mouse with that of a disparate group of other eutherian mammalian species, such as hamster, human, and the bonnet monkey, it was found that this region has a high level of sequence divergence when compared with the rest of the ZP3 glycoprotein (Wassarman \& Litscher 1995). This finding has led to the proposal that changes in this region may result in 'species specificity' of spermZP binding (Kinloch et al. 1995, Wassarman 1995, 
1999, 2002, Wassarman \& Litscher 1995, Wassarman et al. 1999, 2001, 2004a, Williams et al. 2003, 2006). In addition, there is evidence that this region has undergone adaptive evolution with a few of the sites being under positive selection (Swanson et al. 2001). Recently, when comparing the sequence of exon 7 within several species of the genera Mus (Jansa et al. 2003) and Peromyscus (Turner \& Hoekstra 2006), a similar conclusion was reached, although it has also been suggested that the methodology underlying such conclusions may be prone to result in false positives (Berlin \& Smith 2005).

In a previous study on the ZP3 glycoprotein of three endemic species of Australian murine rodents, we found that they shared an identical sequence within the region encoded by exon 7 , albeit that this sequence differed from that of the mouse and rat (Swann et al. 2002). This study suggested that there was a lack of sequence divergence in and around the putative combining-site for sperm of ZP3, and thus contrasts with the conclusions from the studies on Mus (Jansa et al. 2003) and Peromyscus (Turner \& Hoekstra 2006). However, a subsequent reanalysis of the data on the
Mus species, after removal of Rattus as the outgroup, suggested that no positive selection had occurred (Turner \& Hoekstra 2006).

Here, we expand our previous study by determining the nucleotide and predicted amino acid sequence of the exons 6 and 7 regions of ZP3 in a broad range of nonRattus Australasian (New Guinean and Australian 'old endemic') murine rodents. Previous molecular phylogenetic analyses suggest that this group of rodents is monophyletic and it does not appear to have any close relatives outside Australasia (Lee et al. 1981, Watts \& Baverstock 1995, Strahan 2002). They are only distantly related to Mus, although are clearly part of the radiation of the Old World rats and mice (subfamily Murinae) within the family Muridae. Amongst the Australasian species at least six groupings, or divisions as defined by Musser \& Carleton (2005), are recognized. They are the Pseudomys division which is almost entirely restricted to Australia; the Uromys, Xeromys, and Hydromys divisions each of which has species that occur in both Australia and New Guinea; the Pogonomys division that includes a disparate group of genera that are almost entirely restricted to New Guinea; and the Lorentzimys

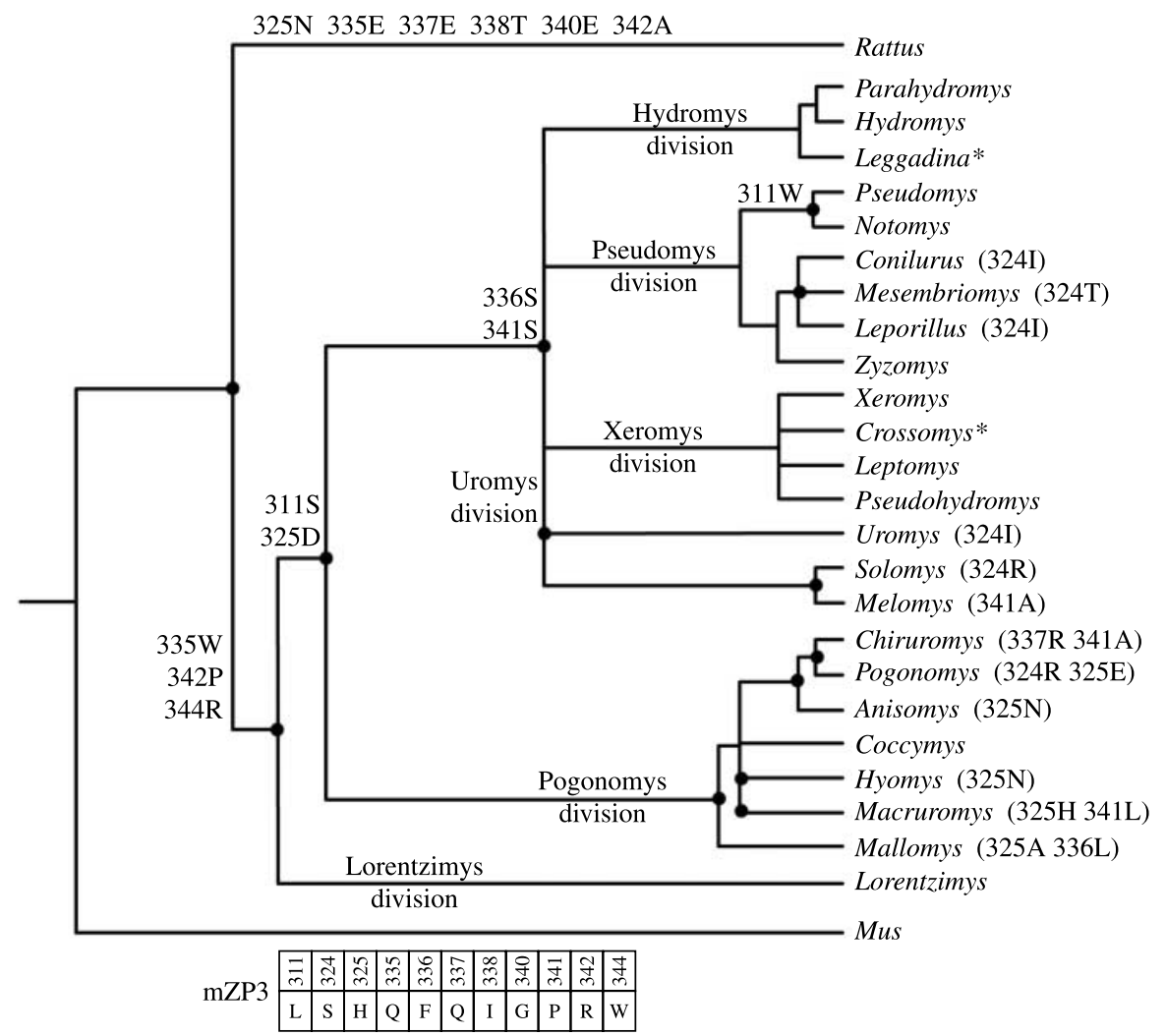

Figure 1 Ancestral reconstruction of the region encoded by exon 7 of ZP3 from the New Guinean and Australasian murines. Proposed phylogeny of the New Guinean and Australasian murines, based on microcomplement fixation of albumin data (Watts \& Baverstock 1995). Amino acid substitutions (single letter code) for selected residue positions for the exon 7 coding regions have been plotted against lineages, with ZP3 from Mus musculus used as reference. Black dots indicate those nodes where amino acid substitutions have taken place. Note the many unresolved branches, and not all genera are represented. The divisions devised by Musser \& Carleton (2005) are noted in red. Under this classification, Crossomys and Leggadina (denoted with an asterisk) have been placed in the Hydromys and Pseudomys divisions respectively. 
division that contains just one species restricted to New Guinea. The first four of these divisions are sometimes placed in a separate subfamily, the Hydromyinae (Watts et al. 1992).

The most comprehensive phylogeny of the New Guinean and Australian murines is one derived from microcomplement fixation of albumin data (see Fig. 1 from Watts \& Baverstock 1995). Recently, a phylogeny of
Australian members of the Pseudomys, Uromys, and Xeromys divisions, based on the nucleotide sequence of the control region of the mitochondrial gene, has been published (see Fig. 2 from Ford 2006). In the present study, these two phylogenies have been used to test for positive selection during the evolution of the $Z p 3$ gene in these species using maximum likelihood analyses. In addition, extensive amino acid sequence comparisons

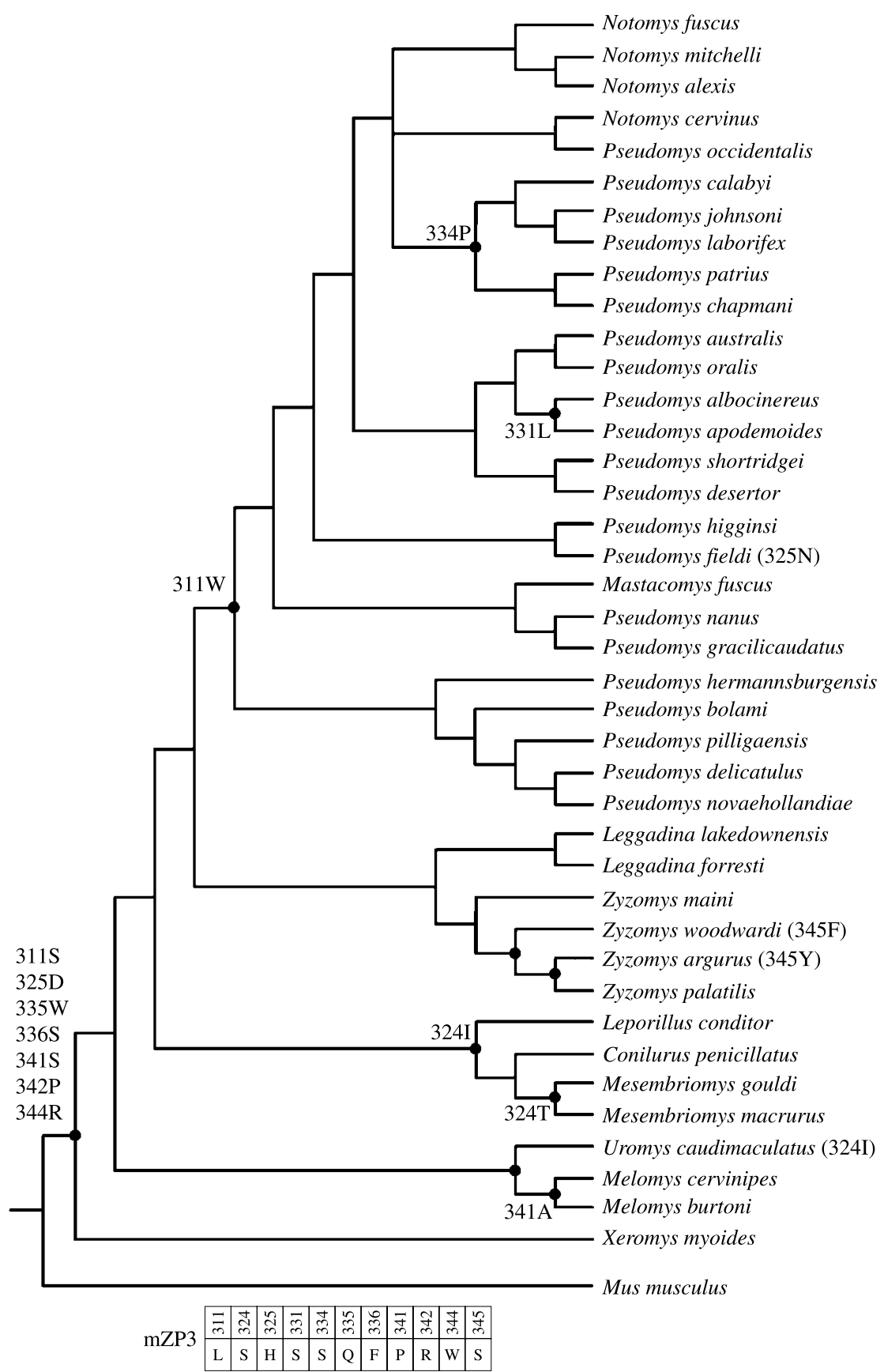

Figure 2 Ancestral reconstruction of the region encoded by exon 7 of ZP3 from the Australian murines. Proposed phylogeny of the Australian murine rodents, based on nucleotide sequence data from the mitochondrial control region (Ford 2006). Amino acid substitutions (single letter code) for seleted residue positions for the exon 7 coding regions have been plotted against lineages, with ZP3 from Mus musculus used as reference only. Black dots indicate those nodes where amino acid substitutions have taken place. 
have been carried out to further investigate whether this region may contribute to species specificity of sperm-ZP binding in these murines.

\section{Materials and Methods}

\section{Nomenclature}

The nomenclature of ZP genes and proteins is confusing since two different systems are in use. Simplification of this nomenclature has been proposed by Harris et al. (1994) and Spargo \& Hope (2002). Recently, Conner et al. (2005) recommended that the numerical system (ZP1-ZP4) based on the size of the ZP glycoproteins when separated by one-dimensional electrophoresis, be adopted, stating that this system has been accepted by the nomenclature committee of the National Center for Biotechnology Information. The recommendation by Conner et al. has been followed in the present study.

\section{Samples and extraction of DNA}

A total of 68 species of New Guinean and Australian murine rodents were included in the study, representing 27 of a total of 35 genera from each of the six divisions as proposed by Musser \& Carleton (2005). DNA was extracted using the PureGene DNA Isolating Kit (Gentra, Minneapolis, MN, USA) from frozen liver tissue provided by the Australian Biological Tissue Collection (ABTC) located at the South Australian Museum. Species names, common names, divisions, ABTC numbers, and GenBank accession numbers for all samples are given in Supplementary Table 1 which can be viewed online at www.reproduction-online.org/supplemental/.

\section{PCR amplification}

Primers were designed using conserved regions of exons 6 and 7 of $Z p 3$ cDNA from the Spinifex Hopping mouse (Notomys alexis; GenBank accession number AY078054; Swann et al. 2002). Forward primer sequence was ACCTGCCATCTCAAAGTCGC and reverse primer sequence was TGCGGTTTCGAGAGGTTAGC. PCR amplifications were carried out in $25 \mu \mathrm{l}$ reaction volumes containing $1 \times$ PCR buffer (Applied Biosystems, Foster, CA, USA), $4 \mathrm{mM} \mathrm{MgCl} 2,0.8 \mathrm{mM}$ dNTPs, $5 \mathrm{pM}$ each primer, $2.5 \mu \mathrm{l}$ of 1:50 diluted DNA, and 0.6 U AmpliTaq Gold DNA polymerase (Applied Biosystems). PCR amplifications were carried out on a Hybaid Omni Thermocycler (Hybaid, Middlesex, UK) at $95^{\circ} \mathrm{C}$ for 9 min; 34 cycles at $94{ }^{\circ} \mathrm{C}$ for $45 \mathrm{~s}, 55^{\circ} \mathrm{C}$ for $45 \mathrm{~s}, 72^{\circ} \mathrm{C}$ for $45 \mathrm{~s}$, and ending with a final elongation cycle at $72{ }^{\circ} \mathrm{C}$ for 6 min. PCR products were then purified using the UltraClean PCR Clean Up Kit (MoBio Laboratories, Solana Beach, CA, USA) following the manufacturer's instructions.

\section{PCR sequencing}

PCR sequencing was performed using the BigDye Sequencing Ready Reaction Kit version 3 (ABI, Perkin Elmer) in $20 \mu \mathrm{l}$ reactions with the PCR primers as sequencing primers. The amount of DNA template used was $5 \mu \mathrm{l}$ per reaction. Reaction products were purified using isopropanol precipitation and sequenced on an $\mathrm{ABI} 3700$ Sequencing Analyser using procedures specified by the manufacturer.

\section{Sequence analysis}

Forward and reverse sequences of each species were compared and manually edited using GeneDoc software (Nicholas et al. 1997) and multiple sequences were then visually aligned. Where the nucleotide sequence between species was identical, repeated extractions of the same species and PCR were performed in order to verify the validity of the sequence.

For comparative purposes, the following Zp3 cDNA sequences were extracted from GenBank: the laboratory mouse (Mus musculus: accession numbers NM_011775) and the laboratory rat (Rattus norvegicus: accession number NM_053762).

\section{Analyses to test for evidence of positive selection}

We first tested for evidence of positive selection by estimating the nonsynonymous (replacement) substitutions per site $\left(d_{N}\right)$ and synonymous (silent) substitutions per site $\left(d_{\mathrm{S}}\right)$ within the exons 6 and 7 regions of $Z p 3$. If $d_{N}$ is less than $d_{S}$, that is the ratio of $d_{N} / d_{S}(\omega)<1$, the gene is considered to be evolving under stabilizing or purifying selection, whereas when $d_{N}=d_{S} \quad(\omega=1)$ the gene is evolving under neutral evolution. A higher $d_{N}$ than $d_{S}$ substitution rate (i.e., $\omega>1$ ) is indicative of positive selection, resulting from nonsynonymous substitutions providing a fitness advantage and being fixed within the population at a higher rate than silent mutations (Yang 1998).

We estimated $d_{\mathrm{N}}$ and $d_{\mathrm{S}}$ using the Yang \& Nielsen (2000) approximation method which allows for transition and base/codon frequency biases. Pairwise comparisons were conducted using the yn00 program of the Phylogenetic Analysis by Maximum Likelihood (PAML) software package (version 3.15; Yang 1997) and mean estimations were calculated over all species, within and between the divisions categorized by Musser \& Carleton (2005). The $\omega$ ratio of $d_{N} / d_{S}$ was not calculated from the $d_{N}$ and $d_{S}$ estimations using this method as the high percentage of $d_{S}=0.000$ produced undefined $d_{N} / d_{S}$ ratios (given as 99 in the PAML software), and would, therefore, introduce bias into the mean $d_{N} / d_{S}$ calculations.

As the above method averaged the $d_{N}$ and $d_{S}$ over all codons, it is not a robust method for detecting evidence 
of positive selection, particularly when it only occurs at a few codon positions, with the majority of sites under stabilizing selection. We, therefore, conducted a maximum likelihood analysis for detecting positive selection using the approach developed by Nielsen \& Yang (1998). This method uses likelihood ratio tests (LRTs) to compare a null model that does not allow for $\omega>1$ and an alternative model which does. We applied three LRTs to the data, using 'site models', rather than 'branch-site' models, as the latter is considered sensitive to small sequences, while the former is not (Yang personal communication). The first pair of models compared was the nearly neutral model (M1a) and the positive selection model (M2a; Wong et al. 2004). The M1a model assumes two codon site classes in proportions $p_{0}$ and $p_{1}=1-p_{0}$ with $0<\omega<1$ and $\omega_{1}=1$. The M2a model adds a proportion $\left(p_{2}\right)$ of sites with $\omega_{2}>1$ estimated from the data. The next pair of models compared was the null M7 model with the alternative model of M8. The M7 model assumes a beta distribution for $\omega$ (within the interval $0<\omega<1$ ) and the M8 model adds an extra class of sites under positive selection $\left(\omega_{\mathrm{s}}>\right.$ 1). The third LRT compared a null model of M8 (M8a) with $\omega_{\mathrm{s}}$ constrained to $1\left(\omega_{\mathrm{s}}=1\right)$ with the M8 model with $\omega_{\mathrm{s}}>1$ (Swanson et al. 2003). For each LRT, the null model is nested within the alternative (positive selection) model so that a test statistic $-2 \Delta \ln L$ (where $\Delta \ln L$ is the difference in log-likelihood values of the two models) follows a $\chi^{2}$ distribution, with the degrees of freedom (df) equal to the difference in the number of parameters between the null and the alternative models. We, therefore, used two df for the M1a/M2a and M7/M8 LRTs and one $\mathrm{df}$ for the M8a/M8 LRT. The correct distribution of the latter test statistic is unknown and, hence, we chose a more conservative one $\mathrm{df}$, rather than using a $\chi^{2}$ distribution with a 50:50 mix of a $\chi_{1}^{2}$ and $\chi^{2}$ with point mass of 0 , a suggested alternative (Wong et al. 2004). All tests are considered to be conservative (Yang et al. 2005). If the LRT is significant $(P<0.05)$ then positive selection can be inferred, and the Bayes Empirical Bayes method (Yang et al. 2005) was then used to calculate the posterior probability (PP) that each codon site is from a particular class of $\omega$. Sites with $\omega>1$ and high posterior probabilities (PP $>95 \%$ ) are considered to be under positive selection.

The implementation of the above site models requires a phylogenetic tree of the species studied. Unfortunately, no published tree, based on nucleotide sequences, currently exists that contains all the taxa in the present study. We, therefore, carried out the analyses using a subset of the taxa and two phylogenies of Australasian murine rodents. The most comprehensive tree of Australasian murine rodents currently available was based on microcomplement fixation of albumin (Watts \& Baverstock 1995; see Fig. 1). This tree contains representative species from most of the New Guinean and Australian old endemic genera. Secondly, we used a recently published phylogenetic tree based on nucleotide data from the mitochondrial control region for 40 Australian species (Ford 2006; see Fig. 2). Sequences used in the analysis are highlighted in Fig. 3. Mus and Rattus data were excluded from the PAML analysis to allow an assessment of whether positive selection occurs specifically within Australasian murine lineages.

\section{Results}

After exclusion of the primer sequences, PCR amplification and sequencing generated fragments of the $Z p 3$ gene between 273 and 305 nucleotides in length. The partial sequences of exons 6 and 7 (171 nucleotides in length) were identified by their similarity to the corresponding cDNA sequence of the mouse and rat $Z p 3$ gene and by the presence of conserved intron/exon splicing signals. Intron 6 varied in length from 102 nucleotides in two Pseudomys species ( $P$. shortridgei and $P$. desertor) to 134 nucleotides in Pseudomys occidentalis.

The coding sequence of exon 6/7 provided a predicted amino acid sequence of 57 residues in length, corresponding to mZP3 glycoprotein Pro-289 to Ser-345 (Fig. 3). Exon 6 of $\mathrm{mZp} 3$ encodes 31 amino acids, of which the last 21 residues have been determined; and exon 7 of $m Z p 3$ encodes 46 amino acids, within which the putative combining-site for sperm occurs, of which the first 36 residues have been determined in the present study.

\section{Protein sequence comparisons}

The results show that, when compared with the mouse (Boja et al. 2003), all five cysteine residues that are potential sites for disulfide bonding (Cys-301, Cys-320, Cys-322, Cys-323, and Cys-328) and all three potential $\mathrm{N}$-linked glycosylation sites (Asn-304, Asn-327, and Asn-330) have been conserved in all species investigated.

\section{Region encoded by exon 6 of Zp3}

All but 9 out of the 68 species investigated have an identical amino acid sequence to that of the mouse and rat (Fig. 3). The exceptions were: in the Uromys division, five Melomys species and Solomys salebrosus, where there was a histidine in position 298; in the Pogonomys division, P. macrourus, with a tyrosine in position 303; and in the Pseudomys division, Notomys cervinus, with a serine residue in position 291 and Zyzomys woodwardi with a valine in position 290.

\section{Region encoded by exon 7 of Zp3}

The five serine residues (Ser-329, Ser-331 to Ser-334) identified in the mouse ZP3 (mZP3) as providing the $O$-linked glycosylation sites (serine/threonine residues) that could be important in sperm-ZP binding are conserved in all but seven species from the Pseudomys division (see below). 
mZP3

Mus musculus Rattus norvegicus Lorentzyimys Division Lorentzimys nouhuysi

Pogonomys Division (1 Anisomys imitator
Chiruromys vates Coccymys ruemmleri Hyomys goliath Macruromys major Mallomys aroaensis Mallomys rothschildi Mammelomys lanosus Mammelomys rattoides Pogonomelomys mayeri Pogonomys macrourus

Hydromys Division (5 g Crossomys moncktoni Hydromys chrysogaster Parahydromys asper

\section{$\begin{array}{cc}289 & 300 \\ * & *\end{array}$ \\ 310 \\ 320 \\ 330 \\ $340 \quad 345$ \\ PANQIPDKLNKACSFNKTSQS WLPVEGDADICDCCSHGNCSNSSSSQFQIHGPRQWS

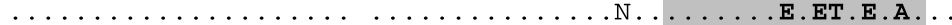

1 genera, 1 species)

3 genera, 25 species)

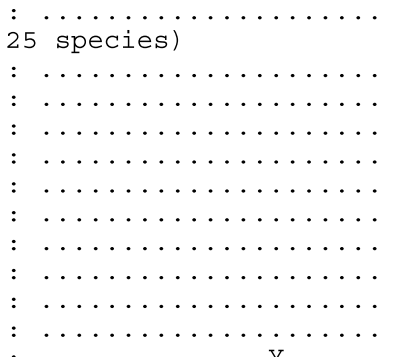

: .................

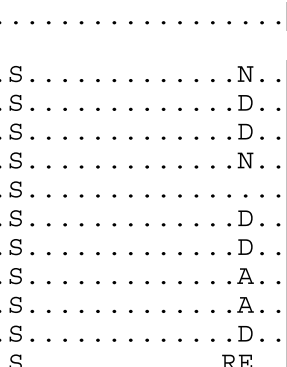

W. . . P.R.

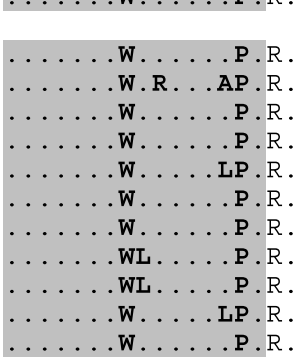

\section{species)}

$: \quad \ldots \ldots \ldots \ldots \ldots \ldots \ldots \ldots \ldots \ldots \ldots \ldots \ldots$

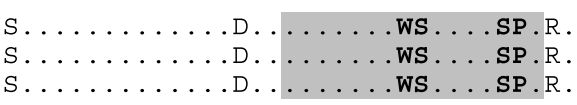

Xeromys Division (3 genera, 8 species)

Leptomys elegans

Pseudohydromys ellermani

Xeromys myoides

Uromys Division (5 genera, 46 species)

Melomys burtoni

Melomys capensis

Melomys cervinipes

Melomys rubicola

Melomys rufescens

Paramelomys levipes

Paramelomys platyops

Paramelomys rubex

Solomys salebrosus

Uromys anak

Uromys caudimaculatus

Pseudomys Division (8 genera,

Conilurus penicillatus

Leggadina forresti

Leggadina lakedownensis

Leporillus conditor

Mesembriomys gouldii

Mesembriomys macrurus

Mastacomys fuscus

Notomys alexis

Notomys aquilo

Notomys cervinus

Notomys fuscus

Notomys mitchellii

Pseudomys albocinereus

Pseudomys apodemoides

Pseudomys australis

Pseudomys bolami

Pseudomys calabyi

Pseudomys chapmani

Pseudomys delicatulus

Pseudomys desertor

Pseudomys fieldi

Pseudomys fumeus

Pseudomys gracilicaudatus

Pseudomys hermannsburgensis

Pseudomys higginsi

Pseudomys johnsoni

Pseudomys laborifex

Pseudomys nanus

Pseudomys novaehollandiae

Pseudomys occidentalis

Pseudomys oralis

Pseudomys patrius

Pseudomys pilligaensis

Pseudomys shortridgei

zyzomys argurus

zyzomys maini

zyzomys palatilis

zyzomys pedunculatus

zyzomys woodwardi

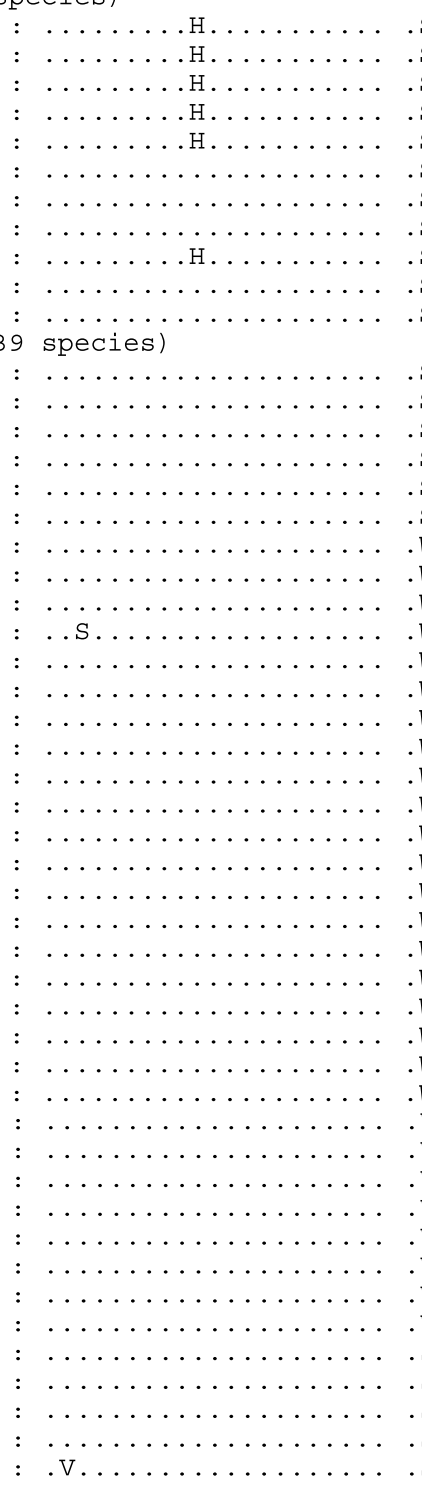

species)
$\quad \ldots \ldots \ldots \ldots \ldots \ldots \ldots \ldots \ldots \ldots \ldots \ldots \ldots \ldots \ldots \ldots$

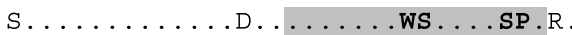

S. . . . . . . . . . . . . WS . R . R

WS....SP.R.

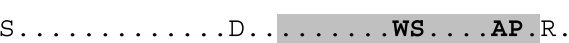

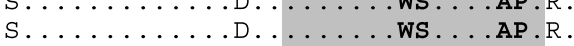

................WSR . . AP.R.

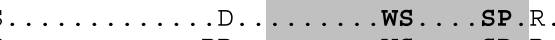

$\ldots \ldots$ RD . . . . . . . . . . RSP .

$\ldots \ldots \ldots$. . . . . . . WS . . . SP.R

$\ldots \ldots \ldots$ D. . . . . . W . . . R . R .

$\ldots \ldots \ldots$. . . . . . . . . . . . .

$\ldots \ldots \ldots$. . . . . . . . . . WS . . SP . R

S...S . . . . ID . . . . . . WS . . SP . R .

S. . . . . . . . . . . . . . . SPS.R.

S. . . . . . . . ID . TWS . . SP.R.

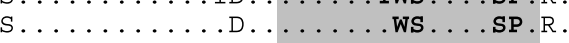

S . . . . . . . . . . . . WS . . R .

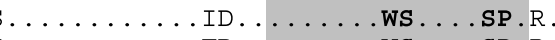

$\ldots \ldots$. . . . . . . . . . . . .

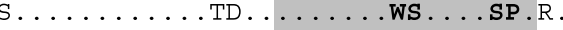

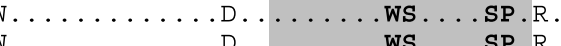

$\ldots \ldots$. . . . . . . . . . . . . . . . .

W. . . . . . . . . . . . . . . . . .

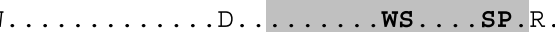

$\ldots \ldots \ldots$. . . . . . . . . . . SPS .

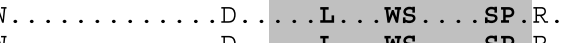

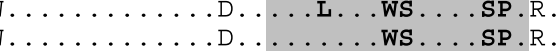

$\ldots \ldots$. . . . . . . . . . . . .

$\ldots \ldots$. . . . . . PWS . . SP.R.

$\ldots \ldots \ldots$. . . . . PWS . . SP . R .

$\ldots \ldots \ldots$ D. . . . . . . . . RS . R .

$\ldots \ldots$. . . . . . . . . . . . .

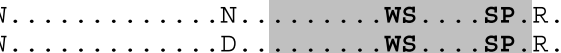

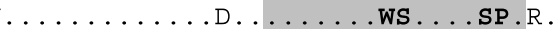

W. . . . . . . . . . . WS . . R .

W. . . . . . . . . . . . W . . . R .

$\ldots$. . . . . . PWS ... SP . R

W. . . . . . . . . . PWS . . R . R .

W. . . . . . . . . . . . WS . . R .

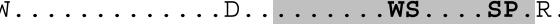

W. . . . . . . . . . . . WS . . R .

W. . . . . . . . . . . . . WS . R . R

W. . . . . . . . . . . . . . . .

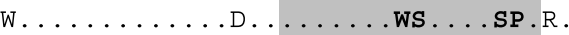

S.......... . . . . . W . . . . SP.RY

S. . . . . . . . . . . WS . . SP.R.

S........ . . . . W . . . SP.R.

$\ldots \ldots$. . . . . . . . . W . . . . SP . R

WS . . . SP.RF 
Lorentzimys division. Lorentzimys nouhuysi is the only species in this division. Three amino acid changes have occurred between the ZP3 sequence from $L$. nouhuysi and the mouse. L. nouhuysi has a tryptophan in position 335 (mZP3: Gln-335), a proline in position 342 (mZP3: Arg-342), and an arginine in position 344 (mZP3: Trp344). None of the amino acid differences that exist between the mouse and the rat are present in the exon 7 region of ZP3 of L. nouhuysi (Fig. 3).

Pogonomys division. The Pogonomys division is composed of 23 extant species (13 genera) of which we have determined the predicted amino acid sequence for 11 species from 9 of the genera. These species differ from the mouse in having Trp-335, Pro-342, and Arg-344. In addition, all species have a serine in position 311 (mZP3: Leu-311). The two species of Mammelomys have an identical amino acid sequence to each other and two species of Mallomys have an identical amino acid sequence to that of Coccymys ruemmleri, albeit different from Mammelomys. Anisomys imitator and Hyomys goliath also share an identical amino acid sequence. Within the ten amino acid residues stretch from 335 to 344 , $54 \%$ ( 6 out of 11) of species in this division share an identical sequence with each other as well as to that of L. nouhuysi, although they differ at these sites from the mouse.

Hydromys and Xeromys divisions. Of the 11 extant species in the Hydromys division and 8 species in the Xeromys division, we determined the predicted amino acid sequence for three species from three genera in both of the two divisions. In all six species, we found an identical sequence. They all share Ser-311, Trp-335, Pro-342, and Arg-344 with species in the Pogonomys division. In addition, all six species have an aspartic acid in position 325 (mZP3: His-325) and serine residues in positions 336 and 341 (mZP3: Phe-336 and Pro-341).

Uromys division. The Uromys division consists of 46 extant species from five genera and we have determined the predicted amino acid sequence from 11 of these species from four genera. All species in this division have, in common with the Hydromys and Xeromys division species, Ser-311, Asp-325, Trp-335, Ser-336, Pro-342 (with one exception being Melomys burtoni), and Arg-344. In addition, in common with species in the Hydromys and Xeromys divisions, all but three Melomys species have a serine residue in position 341. Of the 23 extant Melomys species, we have determined the amino acid sequence in three Australian species (M. burtoni, $M$. capensis, and M. cervinipes), one species from New Guinea (M. rufescens), and the fifth, M. rubicola, from Bramble Cay, a Torres Strait island. The three Australian Melomys species all have an alanine residue in position 341 , which is not present in the other two species of this genus where a serine occurs.

The genus Paramelomys consists of nine species, all endemic to New Guinea, of which we have determined the amino acid sequence for three species. Paramelomys rubex has a serine in position 342, with all other species having a proline in this position. Solomys salebrosus, which is one out of five extant Solomys species endemic to the Solomon Islands, has an identical amino acid sequence to Melomys rufescens with the two species sharing an arginine in position 324 , albeit that the majority of the Australasian murines have a serine in this position. Of the eight extant Uromys species, we have determined the amino acid sequence from two, one of which, Uromys anak occurs only in New Guinea and the other, U. caudimaculatus, occurs in both north-east Australia and New Guinea. U. anak, uniquely, has a serine in position 316 , while other species, including the mouse and rat, have a glutamic acid in this position. Both Uromys species share the common residues with the other members of this division in addition to having an isoleucine residue at position 324, rather than a serine. Within the ten amino acid stretch, from residue 335 to $344,64 \%$ of species from the Uromys division (7 out of 11$)$ shared an identical amino acid sequence.

Pseudomys division. The Pseudomys division comprises 39 extant species, at present allocated to eight genera and we have determined the amino acid sequence from all these species. All species have retained the common Trp335, Pro-342, and Arg-344, which are also shared with species in the other divisions, but not with mouse and rat. In addition, all species have the two additional serine residues at positions 336 and 341 in common with all six species from the Xeromys and Hydromys divisions, and most species from the Uromys division. Furthermore, all but Pseudomys fieldi have an aspartic acid in position 325, in common with all species from Xeromys, Hydromys, and Uromys divisions. All species investigated in the large genus of Pseudomys, together with five species of Notomys and Mastacomys fuscus, have a tryptophan in position 311. All other species in this division (from the genera of Conilurus, Leggadina, Leporillus, Mesembriomys, and Zyzomys), together with species from the Lorentzimys,

Figure 3 Alignment of the predicted amino acid sequence for the region encoded by the partial exons 6 and 7 of Zp3 for New Guinean and Australasian endemic rodent species respectively. Species are grouped under the divisions defined by Musser \& Carleton (2005). In parenthesis, next to each division, are the number of genera per division, and the number of extant species (according to Musser \& Carleton (2005)). Amino acid residues are numbered according to the corresponding mouse ZP3. The exon 6/exon 7 boundary is indicated by the space between residues 309 and 310. The putative combining-site for sperm, identified by Wassarman \& Litscher (1995), is highlighted in grey. Each amino acid is represented by its single letter code: A: (Ala) alanine, C: (Cys) cysteine, D: (Asp) aspartic acid, E: (Glu) glutamic acid, F: (Phe) phenylalanine, G: (Gly) glycine, H: (His) histidine, I: (Ile) isoleucine, K: (Lys) lysine, L: (Leu) leucine, M: (Met) methionine, N: (Asn) asparaine, P: (Pro) proline, Q: (Gln) glutamine, $\mathrm{R}$ : (Arg) arginine, S: (Ser) serine, T: (Thr) threonine, V: (Val) valine, W: (Trp) tryptophan, Y: (Tyr) tyrosine. 
Pogonomys, Hydromys, Xeromys, and Uromys divisions, have a serine in position 311 .

At residue position 324, Conilurus penicillatus and Leporillus conditor have an isoleucine in common with two Uromys species, whereas the two Mesembriomys species have a threonine at this position (Fig. 3). The majority of species also retained the five serine residues (Ser-329, Ser-331 to Ser-334) found in the mouse and rat amino acid sequence. Of the 22 species of Pseudomys investigated two sibling taxa, $P$. albocinereus and $P$. apodemoides, have a leucine in position 331 and five species collectively known as pebble-mound Pseudomys ( $P$. calabyi, P. chapmani, $P$. johnsoni, $P$. laborifex, and $P$. patrius) have a proline in position 334. In addition, Conilurus penicillatus has a threonine in this position. Hence, the five potential $O$-linked glycosylation sites (serine/threonine residues) conserved in the Mus, Rattus, Lorentzimys, Pogonomys, Hydromys, Xeromys, and Uromys divisions have been conserved in all but six of the Pseudomys division species (Fig. 1).

The five Notomys species all share an identical amino acid sequence within the exon 7 coding region, in common with the single Mastacomys species and $64 \%$ of species from the Pseudomys genus. Within the ten amino acid stretch from residue 335 to 344 , all 39 species share the same identical sequence.

\section{Rates of nonsynonymous and synonymous substitutions per site}

Exon 6

Over all six divisions, the mean $d_{\mathrm{S}}$ for exon 6 was 0.041 and $d_{N}$ was 0.006 (Table 1 ). Within each division, $d_{\mathrm{S}}$ was always higher than $d_{N}$, suggesting that exon 6 is evolving under selective constraints (Table 1). Between divisions, the highest mean pairwise comparison of $d_{N}$ was 0.014 between the Pogonomys and the Uromys divisions. Mean pairwise estimations of $d_{\mathrm{S}}$ ranged from 0.016 between the Lorentzimys and the Uromys divisions to 0.057 between the Pogonomys and the Hydromys divisions (Table 1).

\section{Exon 7}

Over all divisions, the mean pairwise estimate of $d_{N}$ for exon 7 was 0.031 (Table 1). The mean pairwise estimate of $d_{\mathrm{S}}$ was 0.037 , a rate similar to that observed for the coding region of exon 6 . Within all divisions, mean pairwise $d_{N}$ estimates were lower than $d_{S}$ estimates, as expected for a sequence under purifying selection (Table 1). However, comparisons between divisions showed a number of cases, where $d_{N}$ was higher than $d_{\mathrm{S}}$ (e.g., Xeromys versus Lorentzimys and Pogonomys; Uromys versus Lorentzimys; Pseudomys versus Lorentzimys; and Pogonomys), suggesting some evidence for

Table 1 Mean estimated pairwise comparisons of nonsynonymous substitutions per nonsynonymous site $\left(d_{N}\right.$, in bold) and synonymous substitutions per synonymous site $\left(d_{S}\right)$ within and between each of the New Guinean and Australasian murine divisions (according to Musser \& Carleton, 2005) for exon 6 (A) and exon 7 (B) coding region of $Z p 3$.

\begin{tabular}{|c|c|c|c|c|c|c|c|c|}
\hline & & \multirow[b]{2}{*}{ Within } & \multicolumn{6}{|c|}{ Between } \\
\hline & & & Lorentzimys & Pogonomys & Hydromys & Xeromys & Uromys & Pseudomys \\
\hline \multicolumn{9}{|c|}{ (A) Exon 6: Overall, mean $d_{N}=0.006, d_{S}=0.041$} \\
\hline \multicolumn{9}{|c|}{$\begin{array}{ll}\text { Lorentzimys } & \boldsymbol{d}_{\mathrm{N}} \\
& d_{\mathrm{S}}\end{array}$} \\
\hline \multirow[t]{2}{*}{ Pogonomys } & & 0.004 & 0.002 & & & & & \\
\hline & $d_{\mathrm{S}}$ & 0.042 & 0.022 & & & & & \\
\hline \multirow[t]{2}{*}{ Hydromys } & $d_{N}$ & 0.000 & 0.000 & 0.002 & & & & \\
\hline & $d_{\mathrm{S}}$ & 0.076 & 0.038 & 0.057 & & & & \\
\hline \multirow[t]{2}{*}{ Xeromys } & $d_{N}$ & 0.000 & 0.000 & 0.002 & 0.000 & & & \\
\hline & $d_{\mathrm{S}}$ & 0.038 & 0.038 & 0.053 & 0.051 & & & \\
\hline \multirow[t]{2}{*}{ Uromys } & $d_{N}$ & 0.012 & 0.012 & 0.014 & 0.012 & 0.012 & & \\
\hline & $d_{\mathrm{s}}$ & 0.034 & 0.016 & 0.037 & 0.049 & 0.044 & & \\
\hline \multirow[t]{2}{*}{ Pseudomys } & $d_{N}$ & 0.002 & 0.001 & 0.003 & 0.001 & 0.001 & 0.013 & \\
\hline & $d_{\mathrm{S}}$ & 0.041 & 0.023 & 0.043 & 0.053 & 0.046 & 0.036 & \\
\hline \multicolumn{9}{|c|}{ (B) Exon 7: Overall, mean $d_{N}=0.031, d_{S}=0.037$} \\
\hline \multicolumn{9}{|c|}{$\begin{array}{ll}\text { Lorentzimys } & \boldsymbol{d}_{\mathrm{N}} \\
& d_{\mathrm{S}}\end{array}$} \\
\hline \multirow[t]{2}{*}{ Pogonomys } & $d_{N}$ & 0.029 & 0.037 & & & & & \\
\hline & $d_{\mathrm{S}}$ & 0.049 & 0.062 & & & & & \\
\hline \multirow[t]{2}{*}{ Hydromys } & $d_{N}$ & 0.000 & 0.055 & 0.042 & & & & \\
\hline & $d_{\mathrm{S}}$ & 0.041 & 0.055 & 0.050 & & & & \\
\hline \multirow[t]{2}{*}{ Xeromys } & $d_{N}$ & 0.000 & 0.055 & 0.042 & 0.000 & & & \\
\hline & $d_{\mathrm{S}}$ & 0.000 & 0.033 & 0.028 & 0.020 & & & \\
\hline \multirow[t]{2}{*}{ Uromys } & $d_{N}$ & 0.025 & 0.065 & 0.051 & 0.014 & 0.014 & & \\
\hline & $d_{\mathrm{S}}$ & 0.043 & 0.037 & 0.055 & 0.043 & 0.025 & & \\
\hline \multirow{2}{*}{ Pseudomys } & $d_{N}$ & 0.017 & 0.060 & 0.058 & 0.016 & 0.015 & 0.029 & \\
\hline & $d_{\mathrm{S}}$ & 0.030 & 0.049 & 0.041 & 0.036 & 0.015 & 0.041 & \\
\hline
\end{tabular}


positive selection acting on the exon 7 region for these lineages. This is particularly evident when mean pairwise comparisons of $d_{N}$ are made between the New Guinean divisions of Lorentzimys and Pogonomys and the other four divisions.

\section{LRTs of positive selection}

To further assess the evidence for positive selection using a statistical approach, we conducted LRTs on two sets of data: Lorentzimys and Pogonomys divisions with some representative species from the other divisions of Hydromys, Xeromys, Uromys, and Pseudomys, using the Watts \& Baverstock (1995) phylogeny (Fig. 1); and Australian species only, using the Ford (2006) mitochondrial control region phylogeny (Fig. 2). Results are shown in Table 2.

LRTs, using representative species from all six divisions (using the Watts \& Baverstock 1995 phylogeny), provided strong evidence of positive selection (Table 2A). In all three tests, the null model of no positive selection was rejected $(P<0.01)$ in favor of the alternative model, i.e., the model of positive selection fits the data significantly better than the null model. Models M2a and M8 identified $12 \%$ of codons as being under strong positive selection $\left(\omega_{\mathrm{s}}=3.57\right)$. Of these seven codon sites, two amino acid sites, mZP3-324 and mZP3-325, gave a PP $>95 \%$ for all models, with a third site identified only under the M8 model (mZP3-341) with a PP $>95 \%$. All three sites are within the exon 7 coding region, although only the first two are close to, but not within, the sequence of residues identified by Wassarman \& Litscher (1995) as the combining-site for sperm. Position 324 contains a serine residue in the majority of species, but this residue was replaced with an arginine in one species in the Pogonomys division, and with either an arginine (two species) or an isoleucine (two species) within the Uromys division. This common serine residue was substituted with either an isoleucine residue (two species) or a threonine (two Mesembriomys species) within the large Pseudomys division. The second site identified as being under positive selection, position 325, changed from an aspartic acid in the majority of species to either an asparagine, alanine, or glutamic acid within the Pogonomys division, but within other divisions, only one species did not have an aspartic acid in this position. Residue mZP3-341 contains a proline in the majority of Lorentzimys and Pogonomys division species, with substitutions to an alanine or a leucine occurring. Within the other four divisions, the majority of species, including the New Guinea Melomys species, have a serine in this position with three Australian Melomys species having an alanine. Each of the above three sites shows parallel site changes, where the same amino acid has independently evolved in different rodent lineages (Fig. 3).

The LRTs using a phylogeny containing 40 species from three divisions of mainly Australian species

Table 2 Likelihood ratio tests of models of codon substitution using PAML (version 3.15).

$2 \Delta \ell$

\begin{tabular}{|c|c|c|c|c|c|c|c|c|c|}
\hline $\begin{array}{l}\text { Mod- } \\
\text { el }\end{array}$ & $\ell$ & M1a-M2a & M7-M8 & M8a-M8 & df & $\boldsymbol{P}\left(\chi^{2}\right)$ & $\begin{array}{l}\omega \text { for each } \\
\text { branch }\end{array}$ & Parameter estimates & $\begin{array}{c}\text { Positively selected } \\
\text { sites (PP >95\%, PP> } \\
99 \% *)\end{array}$ \\
\hline \multicolumn{10}{|c|}{$\begin{array}{l}\text { (A) New Guinea and Australasian species (using combined phylogenetic tree of Watts \& Baverstock (1995) derived from microcomplement fixation } \\
\text { of albumin) }\end{array}$} \\
\hline M1a & -487.39 & & & & & & 0.22 & $\begin{array}{l}p_{0}=0.79, p_{1}=0.21 \\
\omega_{0}=0.011, \omega_{1}=1\end{array}$ & None \\
\hline $\mathrm{M} 2 \mathrm{a}$ & -482.69 & 9.4 & & & 2 & 0.009 & 0.49 & $\begin{array}{l}p_{0}=0.88, p_{2}=0.12 \\
\omega_{0}=0.06, \omega_{1}=1, \omega_{2}=3.57\end{array}$ & $324 \mathrm{~S}, 325 \mathrm{H}$ \\
\hline M7 & -487.45 & & & & & & 0.21 & $p=0.005, q=0.018$ & None \\
\hline M8 & -482.71 & & 9.48 & & 2 & 0.008 & 0.49 & $\begin{array}{l}p=6.43, q=99, p_{0}=0.88, p_{\mathrm{s}}=0.12 \\
\omega_{\mathrm{s}}=3.57\end{array}$ & $324 \mathrm{~S}^{*}, 325 \mathrm{H}^{*}, 341 \mathrm{P}$ \\
\hline M8a & -487.39 & & & 9.36 & 1 & 0.002 & 0.22 & $\begin{array}{l}p=1.15, q=99, p_{0}=0.79, p_{\mathrm{s}}=0.21 \\
\omega_{\mathrm{s}}=1\end{array}$ & None \\
\hline \multicolumn{10}{|c|}{ (B) Australasian species only (using phylogenetic tree, based on data derived from the mitochondrial control region Ford (2006)) } \\
\hline M1a & -458.67 & & & & & & 0.22 & $\begin{array}{l}p_{0}=0.85, p_{1}=0.15, \omega_{0} \& 262 \#= \\
0.076, \omega_{1}=1\end{array}$ & None \\
\hline $\mathrm{M} 2 \mathrm{a}$ & -458.66 & 0.02 & & & 2 & 0.99 & 0.22 & $\begin{array}{l}p_{0}=0.87, p_{2}=0.13, \omega_{0}=0.08, \omega_{1}=1, \\
\omega_{2}=1.13\end{array}$ & None \\
\hline M7 & -458.83 & & & & & & 0.21 & $p=0.18, q=0.66$ & None \\
\hline M8 & -458.66 & & 0.34 & & 2 & 0.84 & 0.22 & $\begin{array}{l}p=8.20, q=99 \\
p_{0}=0.87, p_{\mathrm{s}}=0.13, \omega_{\mathrm{s}}=1.13\end{array}$ & None \\
\hline M8a & -458.67 & & & 0.02 & 1 & 0.88 & 0.22 & $\begin{array}{l}p=8.30, q=99 \\
p_{0}=0.85, p_{\mathrm{s}}=0.15, \omega_{\mathrm{s}}=1\end{array}$ & None \\
\hline
\end{tabular}

Notes: In the above tables, $\ell$, log likelihood ratio: $2 \Delta \ell(-2 \Delta \ln L)=$ twice the difference between log likelihood ratios; df, degrees of freedom; $P\left(\chi^{2}\right)=P$ values of LRT under $\chi^{2}$ distribution, significant values $(<0.05) ; \omega=d_{N} / d_{S}$, for each branch, calculated under each model; $p$, parameters; $\mathrm{PP}$, posterior probability, calculated under Bayes Empirical Bayes method. 
(Xeromys, Uromys, and Pseudomys divisions; Ford 2006), failed to show support for positive selection occurring within this sequence. All three LRTs consistently failed to reject the null model in favor of the positive selection model $(P>0.05)$. The average $\omega$ value across the tree was either 0.21 or 0.22 and, in models allowing for a proportion of sites to have $\omega>1$, the parameter estimate for $\omega$ was 1.13 , which is close to the ratio expected under a neutral model of evolution. In addition, only one residue (mZP3-334) was identified as being under possible positive selection, but the Bayesian PP was $<70 \%$ (Table 2B).

\section{Discussion}

In the mouse, there is good evidence that a region encoded by exon 7 of the Zp3 gene of the extracellular ZP complex acts as the primary binding site for spermatozoa (Kinloch et al. 1992, 1995, Rosiere \& Wassarman 1992, Liu et al. 1995, Wassarman et al. 1997, Chen et al. 1998, Williams et al. 2003, 2006) and cross-species comparisons, using a disparate group of mammals from several orders, have shown that this region of the $Z p 3$ gene exhibits a high degree of sequence divergence and evidence of adaptive evolution (Swanson et al. 2003). This has lead, in part, to the suggestion that in mammals, this sequence divergence may contribute to species specificity of sperm-ZP binding (Kinloch et al. 1995, Wassarman 1995, 1999, 2002, Wassarman \& Litscher 1995, Wassarman et al. 1999, 2001, 2004a, Williams et al. 2003, 2006) and that some of the sites either in, or close to, this region are under positive selection (Swanson et al. 2001, Swanson \& Vacquier 2002, Jansa et al. 2003, Turner \& Hoekstra 2006).

In the present study, we have used the old endemic rodents of New Guinea and Australia to determine the extent of the divergence of exons 6 and 7 of $Z p 3$. We have found evidence for positive selection within exon 7 , but not exon 6, of the Zp3 gene for species within this clade. The amino acid sites where positive selection was found to have occurred (324, 325, and 341) are close to, but not identical with, those found to be under positive selection in the studies using species of Mus by Jansa et al. (2003) and Peromyscus by Turner \& Hoekstra (2006). Two of the sites (324 and 325) are located just outside, and the third site (341) within the combiningsite for sperm as defined by Wassarman \& Litscher (1995). Each of these sites also showed evidence of parallel or convergent evolution of a number of amino acid changes, a finding that can be interpreted as further evidence for adaptive evolution (Briscoe 2001).

Evidence for positive selection was also suggested when the average $d_{N}$ and $d_{S}$ values were compared, with mean $d_{N}$ values greater than $d_{S}$ for pairwise comparisons between species in the two New Guinean divisions, Lorentzimys and Pogonomys, and those in the other divisions. However, when a maximum likelihood analysis was performed on data from species in the Hydromys, Xeromys, Uromys, and Pseudomys divisions, no evidence of positive selection was found for either exons 6 or 7 . This finding is, perhaps, not too surprising in the light of the fact that there are only a few amino acid differences in the sequence of ZP3 for these species. For example, we found an identical amino acid sequence for the region encoded by exon 6 as well as exon 7 of $Z p 3$ in all six species in the Hydromys and Xeromys divisions, three species in the Uromys division, and in four of the species in the Pseudomys division. Average $d_{N}$ was lower than $d_{S}$ in all these groups.

The above findings suggest that positive selection of exon 7 may have only taken place in the lineages leading to extant taxa within the Pogonomys and Lorentzimys divisions and has probably not occurred during the evolution of the exon 7 region of $Z p 3$ in the other lineages. A failure to detect evidence for positive selection among the Australian taxa could possibly be due to a lack of power of the analyses when closely related species are compared and/or due to low sequence variation (Anisimova et al. 2001). However, mean pairwise $d_{S}$ values between these divisions $(0.03)$ were only marginally less than that of the overall mean (0.037) of $d_{\mathrm{S}}$ between all the divisions, including the New Guinea lineages. In contrast to our findings, the recent study of the $Z p 3$ gene of Peromyscus species by Turner \& Hoekstra (2006) showed considerable amino acid sequence variation in the region encoded by exon 7 both between, and even within, the species they studied. Amino acid variation was also found to vary across lineages with some showing multiple substitutions, whilst other lineages had comparable synonymous divergence levels with little, or no, amino acid variation (Turner \& Hoekstra 2006). The latter result is similar to our finding of lineage-specific differences using the Australasian murine rodents.

Although there is ongoing debate, as to whether the spermatozoon binds either to the oligosaccharides on the ZP3 (Wassarman et al. 2004a, 2004b, Williams et al. 2006) or to the supramolecular structure of the ZP matrix (Dean 2003, Rankin et al. 2003, Hoodbhoy \& Dean 2004, Clark \& Dell 2006) in the mouse, there is strong evidence that the region encoded by exon 7 of $\mathrm{mZp3}$ is involved in sperm-ZP binding. Changes to the primary structure adjacent to the combining-site for sperm may alter ionic interactions and/or glycosylation patterns, and hence affect sperm-binding capacity of this molecule. It is possible that, as a result of divergence in the amino acid sequence, the sperm-ZP binding region becomes refractory to the binding of sperm from other species regardless of how this takes place. To prevent cross-species fertilizations, sequence divergence in this region might occur between closely related species that are present in sympatry in parts of their ranges. However, our data 
have shown an identical amino acid sequence in the region encoded by exon 7 , including the residues 335-345, in three pairs of such species in the Australian Pseudomys division: those of Notomys alexis and N. mitchelli, Pseudomys hermannsburgensis and P. bolami, and the two Leggadina species. This finding suggests that, at least within this group of Australian old endemic rodents, positive selection for divergence of exon 7 of $Z p 3$ has not taken place in spite of the occurrence of sympatry between these pairs of species.

Swanson et al. (2003) have suggested that, where divergence of reproductive proteins has taken place, this may have been driven by either sperm conflict and/or sperm competition. In comparative studies of eutherian mammals, relative testis size has been shown to relate to the intensity of potential intermale sperm competition with species that have a polyandrous mating system, and hence exhibiting intense sperm competition, having larger relative testes mass than those where this is not the case (Harcourt et al. 1981, Kenagy \& Trombulak 1986). The Australian endemic rodents have a very large range of relative testes sizes across species. For instance, within the Pseudomys division, most Notomys have relative testes masses of only $0.1-0.2 \%$, and in P. shortridgei and $P$. novaehollandiae, it is $0.35-0.45 \%$ of body mass. By contrast, in other Pseudomys species, for example, P. australis, P. desertor, P. fumeus, P. nanus, and Mastacomys fuscus, relative testis mass is between 1.4 and $3.6 \%$ of body weight (Breed \& Taylor 2000). In the present study, a comparison of exons 6 and 7 coding regions of these species does not show any association between testis size and variation in the combining-site for sperm of the ZP3 glycoprotein. These comparative observations support the recent conclusion from Peromyscus (Turner \& Hoekstra 2006) that there is not an association between adaptive evolution of $Z p 3$ and the potential for intermale sperm competition.

In conclusion, our data provide independent support from the old endemic Australasian murine rodents that positive selection occasionally takes place in the exon 7 region of the $Z p 3$ gene. That this region of $Z p 3$ has undergone adaptive evolution adds further support to the conclusions based on data from Mus and Peromyscus. However, within the Australasian murines, the occurrence of positive selection appears to be restricted to just a few lineages. Most of these species share an identical amino acid sequence between closely related species with no evidence of positive selection having taken place. This low rate of sequence divergence within the lineages of the Australasian murine rodents suggests that it is unlikely that divergence in this region has contributed to species specificity of sperm-ZP binding, if it exists, in these animals.

\section{Acknowledgements}

The authors would like to thank Terry Bertozzi from the South Australian Museum and Fred Ford from James Cook University, Townsville, Queensland for providing tissue and DNA samples, and Kathy Saint from the South Australian Museum for providing technical assistance. In particular, we would especially like to thank Rory Hope for his support, encouragement, and helpful comments during the research for, and preparation of, this manuscript.

\section{Funding}

This work was funded by a Postgraduate Research Scholarship from the Faculty of Health Sciences University of Adelaide, Adelaide, South Australia and a Faculty of Health Science Small Research grant. The authors declare that there is no conflict of interest that would prejudice the impartiality of this scientific work.

\section{References}

Anisimova M, Bielawski JP \& Yang Z 2001 Accuracy and power of the likelihood ratio test in detecting adaptive molecular evolution. Molecular, Biology and Evolution 18 1585-1592.

Berlin S \& Smith NGC 2005 Testing for adaptive evolution of the female reproductive protein ZPC in mammals, birds and fishes reveals problems with the M7-M8 likelihood ratio test. BMC Evolutionary Biology 565.

Bleil JD \& Wassarman PM 1983 Sperm-egg interactions in the mouse: sequence of events and induction of the acrosome reaction by a zona pellucida glycoprotein. Developmental Biology 95 317-324.

Boja ES, Hoodbhoy T, Fales HM \& Dean J 2003 Structural characterization of native mouse zona pellucida proteins using mass spectrometry. Journal of Biological Chemistry 278 34189-34202.

Breed WG \& Taylor J 2000 Body mass, testes mass and sperm size in murine rodents. Journal of Mammalogy 81 758-768.

Briscoe AD 2001 Functional diversification of lepidopteran opsins following gene duplication. Molecular, Biology and Evolution 18 2270-2279.

Chen J, Litscher E \& Wassarman PM 1998 Inactivation of the mouse sperm receptor, mZP3, by site-directed mutagenesis of individual serine residues located at the combining site for sperm. PNAS 95 6193-6197.

Clark GF \& Dell A 2006 Molecular models for murine sperm-egg binding. Journal of Biological Chemistry 281 13853-13856.

Conner SJ, Lefievre L, Hughes DC \& Barratt CLR 2005 Cracking the egg: increased complexity in the zona pellucida. Human Reproduction 20 1148-1152.

Dean J 2003 Reassessing the molecular biology of sperm-egg recognition with mouse genetics. BioEssays 26 29-38.

Ford F 2006 A splitting headache: relationships and generic boundaries among Australasian murids. Biological Journal of the Linnean Society 89 119-138.

Green DPL 1997 Three-dimensional structure of the zona pellucida. Reviews of Reproduction 2 147-156.

Harcourt AH, Harvey PH, Larson SG \& Short RV 1981 Testis weight, body weight and breeding system in primates. Nature 293 55-57.

Harris JD, Hibler DW, Fontenot GK, Hsu KT, Yurewicz EC \& Sacco AG 1994 Cloning and characterization of zona pellucida genes and cDNAs from a variety of mammalian species: the $Z P A, Z P B$ and $Z P C$ gene families. DNA Sequence - The Journal of Sequencing and Mapping 4 361-393.

Hoodbhoy T \& Dean J 2004 Insights into the molecular basis of spermegg recognition in mammals. Reproduction 127 417-422. 
Jansa SA, Lundrigan BL \& Tucker PK 2003 Tests for positive selection on immune and reproductive genes in closely related species of the murine genus Mus. Journal of Molecular Evolution 56 294-307.

Kenagy GH \& Trombulak SC 1986 Size and function of mammalian testes in relation to body size. Journal of Mammalogy 67 1-22.

Kinloch RA, Mortillo S \& Wassarman PM 1992 Transgenic mouse eggs with functional hamster sperm receptors in their zona pellucida. Development 115 937-946.

Kinloch RA, Sakai Y \& Wassarman PM 1995 Mapping the mouse ZP3 combining site for sperm by exon swapping and site-directed mutagenesis. PNAS 92 263-267.

Lee AK, Baverstock PR \& Watts CHS 1981 Rodents - the late invaders. In Ecological Biogeography of Australia, pp 1521-1554. Ed. A Keast. The Hague: Junk.

Liu C, Litscher E \& Wassarman PM 1995 Transgenic mice with reduced numbers of functional sperm receptors on their eggs reproduce normally. Molecular Biology of the Cell 6 577-585.

Musser GG \& Carleton MD 2005 Superfamily Muroidea. In Mammal Species of the World, pp 894-1531. Eds DE Wilson \& DM Reeder. Baltimore, MD: John Hoptkins University Press.

Nicholas KB, Nicholas HBJ \& Deerfield DWI 1997 GeneDoc: analysis and visualization of genetic variation. EMBNET NEWS 414.

Nielsen R \& Yang Z 1998 Likelihood models for detecting positively selected amino acid sites and application to the HIV-1 envelope gene. Genetics 148 929-936.

Rankin T, Familari M, Lee E, Ginsberg A, Dwyer N, BlanchetteMackie J, Drago J, Westphal H \& Dean J 1996 Mice homozygous for an insertional mutation in the Zp3 gene lack a zona pellucida and are infertile. Development 122 2903-2910.

Rankin TL, Coleman JS, Epifano O, Hoodbhoy T, Turner SG, Castle PE, Lee E, Gore-Langton R \& Dean J 2003 Fertility and taxon-specific sperm binding persist after replacement of mouse sperm receptors with human homologs. Developmental Cell 5 33-43.

Rosiere T \& Wassarman PM 1992 Identification of a region of mouse zona pellucida mZP3 that possesses sperm receptor activity. Developmental Biology 154 309-317.

Spargo SC \& Hope RM 2002 Evolution and nomenclature of the zona pellucida gene family. Biology of Reproduction 68 358-362.

Strahan R 2002 The Mammals of Australia, 2nd edn, Sydney: Australian Museum, Reed New Holland.

Swann CA, Hope RM \& Breed WG 2002 cDNA nucleotide sequence encoding the ZPC protein of Australian hydromyine rodents: a novel sequence of the putative sperm-combining site within the family Muridae. Zygote 10 291-299.

Swanson WJ \& Vacquier VD 2002 The rapid evolution of reproduction proteins. Nature Reviews Genetics 3 137-144.

Swanson WJ, Yang Z, Wolfner MF \& Aquadro CF 2001 Positive Darwinian selection drives the evolution of several female reproductive proteins in mammals. PNAS 98 2509-2514.

Swanson WJ, Nielsen R \& Yang Q 2003 Pervasive adaptive evolution in mammalian fertilization proteins. Molecular Biology and Evolution 20 18-20.

Turner LM \& Hoekstra HE 2006 Adaptive evolution of fertilization proteins within a genus: variation of ZP2 and ZP3 in deer mice (Peromyscus). Molecular, Biology and Evolution 23 1656-1669.

Wassarman PM 1995 Towards molecular mechanisms for gamete adhesion and fusion during mammalian fertilization. Current Opinion in Cell Biology 7 658-664.

Wassarman PM 1999 Mammalian fertilization: molecular aspects of gamete adhesion, exocytosis and fusion. Cell 96 175-183.

Wassarman PM 2002 Sperm receptors and fertilization in mammals. Mount Sinai Journal of Medicine 69 148-155.
Wassarman PM \& Litscher ES 1995 Sperm-egg recognition mechanisms in mammals. Current Topics in Development Biology 30 1-19.

Wassarman PM, Qi H \& Litscher E 1997 Mutant female mice carrying a single mZP3 allele produce eggs with a thin zona pellucida, but reproduce normally. Proceeding of the Royal Society, London Part B $264323-328$.

Wassarman PM, Chen J, Cohen N, Litscher E, Liu C, Qi H \& Williams Z 1999 Structure and function of the mammalian egg zona pellucida. Journal of Experimental Zoology Part B Molecular and Developmental Biology 285 251-258.

Wassarman PM, Jovine L \& Litscher E 2001 A profile of fertilization in mammals. Nature Cell Biology 3 E59-E64.

Wassarman PM, Jovine L, Litscher E, Qi H \& Williams Z 2004a Eggsperm interactions at fertilization in mammals. European Journal of Obstetrics, Gynecology and Reproductive Biology 115 S57-S60.

Wassarman PM, Jovine L \& Litscher ES 2004b Mouse zona pellucida genes and glycoproteins. Cytogenetic and Genome Research 105 228-234.

Watts CHS \& Baverstock PR 1995 Evolution in the murinae (Rodentia) assessed by microcomplement fixation of albumin. Australian Journal of Zoology 43 105-118.

Watts CHS, Baverstock PR, Birrell J \& Kreig M 1992 Phylogeny of the Australian rodents (Muridae): a molecular approach using microcomplement fixation of albumin. Australian Journal of Zoology $\mathbf{4 0}$ 81-90.

Williams Z, Litscher E \& Wassarman PM 2003 Conversion of Ser to Thr residues at the sperm combining-site of mZP3 does not affect sperm receptor activity. Biochemical and Biophysical Research Communications $301813-818$.

Williams Z, Litscher E, Jovine L \& Wassarman PM 2006 Polypeptide encoded by mouse ZP3 exon-7 is necessary and sufficient for binding of mouse sperm in vitro. Journal of Cellular Physiology 207 30-39.

Wong WS, Yang Z, Goldman N \& Nielsen R 2004 Accuracy and power of statistical methods for detecting adaptive evolution in protein coding sequences and for identifying positively selected sites. Genetics 168 1041-1051.

Yanagimachi R 1994 Mammalian Fertilization. In The Physiology of Reproduction, 5 edn, pp 189-317. Eds E Knobil \& JD Neill. New York: Raven Press.

Yang Z 1997 PAML: a program package for phylogenetic analysis by maximum likelihood. Computer Applications in the Biosciences 13 555-556.

Yang Z 1998 Likelihood ratio tests for detecting positive selection and application to primate lysozyme evolution. Molecular, Biology and Evolution 15 568-573.

Yang Z \& Nielsen R 2000 Estimating synonymous and nonsynonymous substitution rates under realistic evolutionary models. Molecular Biology and Evolution 17 32-43.

Yang Z, Wong WS \& Nielsen R 2005 Bayes empirical bayes inference of amino acid sites under positive selection. Molecular, Biology and Evolution 22 1107-1118.

Received 25 May 2006

First decision 20 June 2006

Revised manuscript received 17 December 2006

Accepted 10 January 2007 